\title{
Two-choice, observational learning and reversal in the rat: S-S versus S-R effects
}

\author{
M. RAY DENNY, R. CHARLES BELL, and CARLA CLOS \\ Michigan State University, East Lansing, Michigan
}

\begin{abstract}
In Experiment 1, male rats were trained to press both bars in a two-choice apparatus and were then given observational training of a go/no-go discrimination in which the observed operation of two inaccessible, dissimilar bars by a hidden experimenter constituted S+ and S-. After discrimination was established, individual rats were permitted access to the two bars. Six of the seven rats consistently pressed the $S+$ bar on 10 test trials, but failed to reverse bar preference after observational training was reversed. In Experiment 2, nine naive males received the same observational training as in Experiment 1, but without any pretraining to press either bar. All rats pressed the S+ bar on initial test and did so consistently throughout the 10 trials. Six of these rats received reversal training of the go/no-go discrimination after the 10 test trials. As in Experiment 1, all rats failed to press the new S+ bar. However, five of six rats in another group, which received reversal training prior to any test trials, did reverse and press the new S+ bar. In Experiment 3, controls for possible confounding effects of overtraining trials were conducted. These manipulations had no effect; the rats tested before reversal still failed to press the $S+$ bar, and the rats reversed before testing all reversed or pressed the most recent $S+$ bar. That is, S-R learning predominated over S-S learning if active, though unreinforced, responding to a particular bar intervened. In contrast, however, a cognitive (S-S) interpretation of directed response learning was supported by the results of Experiment 4, in which the rats that learned the go/no-go discrimination without responding (only by auditory and light cues) failed to press the $\mathbf{S}+$ bar consistently.
\end{abstract}

Over the years it has become increasingly clear that the response-reinforcer contingency is not necessary for learning an instrumental response (Lajoie \& Bindra, 1976). As these investigators point out, "phenomena of response substitution (Lashley \& McCarthy, 1926), observational learning (Kohn \& Dennis, 1972), and learning without responding (Dodwell \& Bessant, 1960; Solomon \& Turner, 1962) demonstrate that a response can be acquired without its reinforcement or even its occurrence during the training trials"' (p. 157).

In the present study, the rat's observation of distinctive environmental events with definite hedonic consequences (stimulus sequences) does lead to the learning of the appropriate response in the absence of its occurrence. The rat first watches both positive and negative events occur, and demonstrates go/ no-go discrimination that indicates it has learned these S-S relationships. The learning of the appropriate response is then indexed by permitting the animal to make either the response that should produce the positive stimulus event or the one that should produce the negative event. That an observation-trained rat makes the appropriate response

This research was supported, in part, by All University Research grants. Requests for reprints should be directed to: $M$. R. Denny, Department of Psychology, Michigan State University, East Lansing, Michigan 48824. when permitted to do so fits a cognitive, or S-S, view of learning, somewhat reminiscent of Tolman's (1932) "what leads to what" theory and largely in contrast to the results of the Spence and Lippitt (1946) latent learning studies. Here it should be emphasized that the old latent learning studies tested for S-S relations that were irrelevant to the rat's motivational state, whereas the present study is concerned with S-S learning that is relevant.

In part, the present study can be conceptualized as contributing to an analysis of standard observational learning (Del Russo, 1975; Groesbeck \& Duerfeldt, 1971; Kohn \& Dennis, 1972). Three cues can be identified in the standard observational learning procedure: the manipulandum, some movement or change in the manipulandum, and the model. It may well be, however, that the model is a relatively unimportant factor. The observation of changes in the manipulandum in correlation with reinforcement or nonreinforcement may be sufficient to elicit a full-blown directed response in subsequent test trials, as found by Jacobson and Sisemore (1976) in human subjects.

The standard procedure that evolved at the completion of Experiment 1 used completely naive rats, ones that had never pressed a bar (manipulandum) before. After these rats had learned a go/no-go discrimination through observing that the experimenterproduced movement of one bar $(\mathrm{S}+)$ was associated 
with obtaining food and the movement of the other bar (S-) was not, they were given access to the bars to see which one, if either, they pressed. Thus, the present study can be classified as being similar to a discriminative autoshaping experiment in which responding to the discriminanda is blocked (Browne, 1976), or as a model-absent observational learning study. Both paradigms share the same elements: the observation of contingencies and a transfer-oftraining test.

\section{EXPERIMENT 1}

The general procedure becomes clear from a diagram of the apparatus used (Figure 1). The rat is stationed in the hardware-cloth tunnel looking out from behind the closed door at the two bars in the test chamber. The hidden experimenter operates the bars individually from behind the opaque end wall, and the operation of one particular bar (S+) is consistently correlated with the presence of food at the other end of the tunnel. After the rat has clearly learned to differentiate between the operation of the two bars, it is permitted to enter the test chamber to see whether it will press the correct bar.

When we first began this research, we seriously doubted that a naive rat that had learned to discriminate between the activations of the two bars just by watching from the tunnel would actually press either bar when they were made accessible during test trials. Consequently, the initial phase of Experiment 1 involved the conventional training of each rat to press both bars about equally well

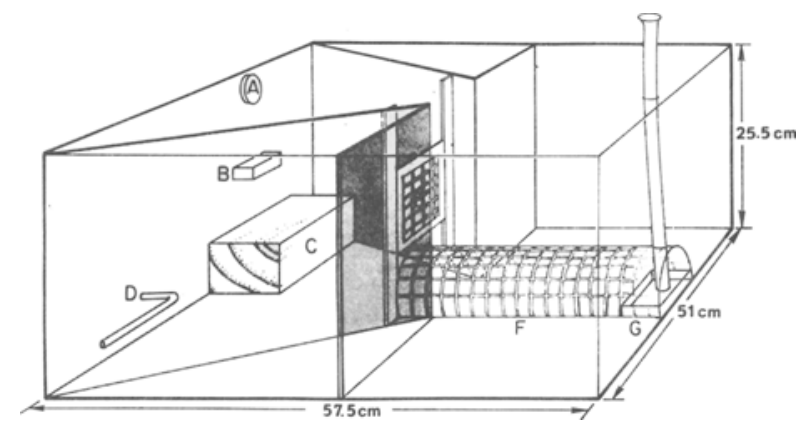

Figure 1. The two-choice observation apparatus. The funnelshaped compartment containing bars is called the test chamber. (A) The light above right bar. (B) Right bar. (C) Wooden block below right bar. (D) Left bar. (E) Hardware-cloth tunnel door in up position, when rat can leave or enter tunnel. Door is down when rat is in tunnel watching bars being activated. (F) Hardwarecloth tunnel in which rat observes bars and jackknifes around to obtain food reward. (G) Food tray in which pellet is dropped through tube. The ceiling over the tunnel is opaque; the one over the bars is transparent, letting in room light. The experimenter, sitting almost opposite to the tip of the left bar, can see the subject at all times through the near wall opposite to the tunnel, but the subject cannot see the experimenter until after it turns toward the food tray. (All other walls are opaque.) before they received observational training in the tunnel. We were simply concerned with whether the rats would press the bar whose operation was uniquely correlated with food.

\section{Method}

Subjects. Seven hooded male rats, approximately 150 days of age and from a colony maintained by the Department of Psychology at Michigan State University, were employed as subjects. Hooded rats were selected because of their superior vision. The rats were maintained at about $90 \%$ of ad-lib weight and were $23 \mathrm{~h}$ deprived of food when run in the experiment.

Apparatus. The two-choice observation enclosure is pictured in Figure 1. The bars moved by the experimenter from behind the opaque end panel had certain stimulus consequences. The tip of the left bar had a total excursion of $8.5 \mathrm{~cm}$ and made a sharp click when it struck the chamber floor. The activated right bar had an excursion of $2.2 \mathrm{~cm}$, turned on a dim light above the bar, and produced a faint click from the microswitch.

Preliminary training. Training the rats to press either bar to receive reinforcement at the end of the tunnel was a laborious task. In nearly every case, the bars had to be baited with wet mash and the wet mash gradually faded. Shaping was also employed. Presumably the delayed reinforcement involved in entering the tunnel and traversing its length to the food tray as well as learning two responses more or less simultaneously made the learning difficult. All rats were trained until they pressed a bar within $10 \mathrm{sec}$ of entering the test chamber, and they were equally often reinforced on each bar. To effect this equation, a rat was occasionally prevented from having access to a particular bar through use of a clear plastic barrier. Each rat was then given a test for bar preference with either 10 or 11 discrete trials; in every case but one, a preference was determined within 10 trials, with a rat selecting one bar either 6 or 7 times. In the observational training that followed, the $S+$ bar was always the nonpreferred bar (four rats trained with $S+$ bar on the right, three with $S+$ bar on left). All rats were quickly pretrained in the tunnel to leave the food tray and approach the tunnel door by being given a $97-\mathrm{mg}$ food pellet each time they did this, for a total of five reinforcements.

Observational training. This phase began on the day following the end of pretraining. Trials occurred only when the rat in the tunnel was looking toward the test chamber (Figure 1). With very few exceptions, the rats promptly turned toward the tunnel door after visiting the food tray. On S+ trials of the go/no-go discrimination, a 97-mg Noyes food pellet was delivered only after the rat had jackknifed around in the tunnel en route to the food tray. This procedure guaranteed that the moving bars and attendant stimuli maintained exclusive stimulus control, of the go/no-go behavior, since the slight noise of the pellet's striking the tray followed the response. Also, the intertrial interval (ITI) was a computer-generated Poisson distribution of time intervals, with a mean of $10 \mathrm{sec}$ and a range of 1 to $60 \mathrm{sec}$, so wait-time could not become a reliable cue for leaving the tunnel door and approaching the food tray. The ITI started after the rat returned to the door from the food tray or after the rat had waited $10 \mathrm{sec}$ at the door on S- trials. Extra visits to the food tray during the ITI increased the ITI only if an animal failed to return to the door by the end of the ITI, and post-ITI returns were never followed by an $S+$ or $S-$ trial until 3 to $5 \mathrm{sec}$ had elapsed.

Trials were administered in blocks of $20,10 \mathrm{~S}+$ and $10 \mathrm{~S}-$ trials randomly distributed; generally, one or two blocks were presented daily until the discrimination criterion was reached. The criterion was (1) inhibiting on 9 of $10 \mathrm{~S}-$ trials by continuing to face the test chamber for at least $10 \mathrm{sec}$ after the $\mathrm{S}-$ bar moved, and (2) leaving the tunnel door for the food tray in less than $10 \mathrm{sec}$ on all $10 \mathrm{~S}+$ trials of a block (well-trained subjects left in 1 or $2 \mathrm{sec}$ ).

As soon as the criterion was reached, the tunnel door was raised, and when the rat entered the test chamber, the tunnel 
door was closed behind it. As soon as a bar was pressed (turning the light on for the right bar and deflecting the left bar to a horizontal position or below from a position .45 rads above horizontal), the rat was removed from the test chamber and returned to its home cage. Thus, with respect to the food tray, the rat was neither reinforced nor nonreinforced for barpressing during the test, although conditioned reinforcement could presumably occur as a result of the light or sharp click. On each of 3 succeeding days, the rat was given 20 more observation trials followed immediately by a test, as described above. On the next 3 days, the rat was tested directly before the 20 booster trials and directly after, yielding a total of 10 test trials.

At the completion of the 10 test trials, all subjects that had shown a clear tendency to press the $\mathbf{S}+$ bar were given reversal training in the tunnel; that is, the original $S+$ bar became the $S-$ bar, and the original S- bar, the new S+ bar. Observational training continued until the same criterion as originally used was met, and then all subjects were administered 10 test trials, as described above. On early $\mathbf{S}+$ trials, in reversal, in which the subject inhibited for $10 \mathrm{sec}$, the $\mathrm{S}+$ bar was reactivated and the rat always received reinforcement on $S+$ trials.

\section{Results and Discussion}

The seven rats learned the go/no-go discrimination in a mean of 232 trials, including the criterial block. Six of them pressed the $S+$ bar on the initial test trial, and those six pressed the $\mathrm{S}+$ bar on an average of $83 \%$ of the 10 test trials. The other rat (trained to right bar as $\mathrm{S}+$ ) pressed the $\mathrm{S}-$ bar $80 \%$ of the time. After reversal training in the tunnel, the six reversed rats persisted in pressing the old $S+$ bar on an average of $85 \%$ of the test trials, even though the go/no-go discrimination reversal was learned in a mean of 210 trials.

On the initial test trial, a rat typically explored the test chamber for a minute or two before pressing a bar (the test chamber was funnel-shaped to minimize exploration), but by the last two test trials barpressing occurred within 3 to $25 \mathrm{sec}$ and the bar was typically depressed the full distance. Performance on the 3 test trials given prior to the 20 extra training trials was not degraded.

These generally positive results encouraged us to eliminate the barpress pretraining in Experiment 2 to see whether observational training alone would produce appropriate responding during test in rats that had never pressed a bar.

\section{EXPERIMENT 2}

We first ran three naive rats with the new procedure and discovered that they would press the $\mathrm{S}+$ bar as well as, if not slightly better than, the rats of Experiment 1. By this time, an explanation of the reversed rats' failure to reverse bar preference during the second test session in Experiment 1 became apparent. So these three rats were used to pilot the critical manipulation. We then designed a two-group experiment to test our hypothesis. The hypothesis involved the following points. Rats learn a particular barpress during test trials through consistent responding in the test chamber stimulus situation (Denny \& Adelman, 1955). This learned response is protected from extinction because reversal training in the tunnel is in a stimulus situation that is different from the situation in which the barpressing occurred. For extinction to take place, the original response and the competing response must occur in similar stimulus situations (Denny, 1971; Denny \& Adelman, 1955). The test of this interpretation was to reverse a group on the go/no-go discrimination before it had received any test trials. Without test trials to mediate S-R learning, any response mediated by the original S-S learning should be extinguished by learning the S-S reversal. Thus, the hypothesis under test is that the new $\mathbf{S}+$ bar is consistently pressed if all test trials follow the reversal training.

To test for generality across strains, both hooded and albino rats were used in Experiment 2.

\section{Method}

Subjects and Apparatus. Seven albino and five hooded male rats, 165 days of age, were divided as equally as possible into two groups of six rats each. Three additional males, two hooded and one albino, were used to pilot the testing procedure. All rats were under 23-h food deprivation and were maintained at $90 \%$ of ad-lib weight because more highly deprived rats bit the tunnel door and visited the food tray excessively often. The apparatus was identical to that of Experiment 1.

Prellminary training. Individual rats explored both halves of the observation apparatus for approximately $10 \mathrm{~min}$, with both bars inoperable. Ten $97-\mathrm{mg}$ Noyes pellets were available in the food tray during exploration. Next, five additional food pellets were individually delivered while the rat was confined to the tunnel; each pellet was delivered only after the rat had left the food tray and faced the closed tunnel door before returning to the food tray (Figure 1).

Observational training. This training, prior to test trials, was identical to Experiment 1 for all 15 rats, except that half of the animals in each group had the left bar as $\mathrm{S}+$ and half had the right bar. For the six rats that were reversed before testing (Group RBT), reversal training began on the day after the criterion was met and continued until this criterion was reached again. All rats received their first test trial on the criterial day; the remaining nine trials were delivered as described for Experiment 1. A second reversal followed the first set of test trials and was carried to the same criterion. A second testing session of 10 trials was then administered as before. The six rats tested before reversal (Group TBR) received all 10 test trials administered as in Experiment 1, that is, with 120 additional observational learning, or booster, trials prior to reversal learning and the final 10 test trials. All test-trial sessions for both groups involved the 120 booster trials. Since the pilot animals that received one or two test trials prior to reversal training performed close to chance on second testing, no test trials at all were given Group RBT before reversal training.

\section{Results and Discussion}

The original go/no-go discrimination was learned in a mean of 207.5 trials $(\mathrm{N}=15)$, the first reversal in a mean of $209.6(\mathrm{~N}=15)$, and the second in a mean of 196.6 trials $(\mathrm{N}=5)$. All nine rats tested before reversal pressed the $\mathrm{S}+$ bar on the initial test trial and pressed the $S+$ bar on an average of $91 \%$ 
of all the test trials. Group TBR pressed the S+ bar on an average of $87 \%$ of the test trials during the first test session and the same bar on an average of $82 \%$ of the test trials after reversal training. That is, as in Experiment 1, all subjects of Group TBR failed to reverse bar preference. Five of the six rats in Group RBT pressed the new S+ bar on an average of $92 \%$ of the 10 test trials, while one rat (hooded) never pressed it. After the second reversal, these same five rats failed to reverse, pressing the new S- bar on an average of $96 \%$ of the 10 test trials. Again, intervening barpressing during test trials, which presumably involved S-R learning, since latencies declined steadily over test trials, prevented reversal of bar preference. With Fisher's exact test, the difference between groups on the 10 test trials following the first reversal was highly significant $(p=.00075)$.

If the data from both Experiments 1 and 2 are combined for the critical initial test trial following the original go/no-go discrimination, 15 of 16 rats pressed the $S+$ bar $(p=.00026$ by the exact binomial test).

In short, albinos showed model-absent observational learning roughly to the same degree as hooded rats, and the hypothesis that S-S reversal learning of bar preference occurs only when intervening barpressing on test is eliminated was supported.

However, since Group TBR had 120 more trials on the original go/no-go discrimination than Group RBT before it received reversal training, there is the possibility that overtraining could have produced the failure of Group TBR to reverse bar preference. This would seem to be very unlikely because overtraining, if anything, typically has the opposite effect, facilitating, rather than hindering, reversal learning. But a direct test of this possibility was in order.

\section{EXPERIMENT 3}

To control for the 120 extra trials' possibly confounding effects on reversal performance, a complete experiment was run. To control this factor in the two ways possible, a group that was reversed before testing also received an additional 120 trials on the original go/no-go discrimination, and a group that was tested before reversal received no extra trials during the initial testing phase.

\footnotetext{
Method

Subjects, Apparatus, and Preliminary Training. Eighteen albino males, approximately 110 days of age, served as subjects. The apparatus and preliminary training were as in Experiment 2.

Observational training. In Experiment 3, there were three groups of six rats each. An extra trials control (ETC) group received 120 overtraining trials, 20 per day, after reaching original criterion. These trials came before the rats were reversed and before any testing. After reversal training and after they
}

reached the reversal criterion, ETC received its initial set of 10 test trials and then was reversed and tested again. This final step indexed the effect of the initial testing period. There were two tested before reversal (TBR) groups, one without the extra training, or booster, trials during the initial testing phase (TBRno extra) and one with the 120 extra training trials as in Experiment 2 (TBR). All trials were administered in the same fashion with the same ITI as in Experiment 2 . Reversal training and subsequent testing were specifically the same as in Experiment 2, that is, with booster trials given during test days for all groups in order to maximize the possibility of reversing bar preference.

\section{Results and Discussion}

All three groups showed similar observational learning, with means to criterion of 196.7, 196.7, and 213.3 on the original go/no-go discrimination and means of $250,216.7$, and 220 on first reversal for ETC, TBR-no extra, and TBR, respectively. In the only group that received a second reversal (ETC), the customary trend toward facilitation was apparent (mean $=156.7$ trials).

All six rats in Group ETC pressed the new S+ bar on the initial test trial, and on the average pressed it $93.3 \%$ of the 10 test trials, clearly showing reversal of the directed response. Thus, the 120 overlearning trials did not prevent reversal. When ETC was reversed for the second time on the go/no-go discrimination, this time after 10 test trials, it failed to reverse bar preferences, as was true of all groups tested before reversal, pressing the new $\mathrm{S}+$ bar on an average of only $10 \%$ of the 10 test trials.

Five of six TBR subjects pressed the $\mathrm{S}+$ bar on the first test trial. However, for the first time in this type of experiment, two male rats in the TBR group responded at chance level to the bars on initial testing, yielding as a group $66.7 \%$ pressing of the $\mathrm{S}+$ bar overall. One of these two animals reversed bar preferences after reversal training, pressing the same bar as the last three barpresses on the initial set of 10 test trials at a $100 \%$ level. The rat that did not reverse also pressed, at a $100 \%$ level on the second test, the same bar as the last three barpresses of initial testing (the last three barpresses happened to be consistently to one particular bar for both subjects). In other words, it appears that three unreinforced barpresses in a row can yield sufficient S-R learning in the test chamber via contiguity or elicitation principles (Denny, 1971; Guthrie, 1952) to mediate consistent responding thereafter (Denny, 1959).

The other four rats in TBR continued to press the original $\mathrm{S}+$ on an average of $97.5 \%$ of the 10 test trials after reversal training, failing like all other TBR groups to reverse bar preference. Likewise, all six rats in the TBR-no extra group pressed the $\mathrm{S}+$ bar on the initial test trial and on $86.7 \%$ of the first set of 10 test trials, and then pressed the old $\mathrm{S}+$ on an average of $95 \%$ of the 10 test trials following reversal training. In short, TBR-no extra 
and TBR, groups that differed only on whether or not they received the 120 extra trials during testing, did not differ significantly on reversal of bar preference (Mann-Whitney U test, $p>.53$ ), while TBR and ETC, which differed only on whether or not they received test trials prior to reversal training, did differ significantly on reversal performance (Mann-Whitney $\mathrm{U}$ test, $\mathrm{p}<.02$ ). Overtraining, or extra trials, is irrelevant for reversal of bar preference.

\section{EXPERIMENT 4}

In addition to controls for the 120 extra trials, there was another important control that needed to be conducted. There was the distinct possibility that a rat in the test chamber simply approached the region where the $\mathbf{S}+$ bar was located and, in so doing, accidentally pressed this bar. It is possible that the rat does not see the bars move during observational training and is not attempting to make the $\mathrm{S}+$ bar move during test. Observation of the rats' behavior during the later stages of observational training indicated, however, that the rats were attending closely to bar movement; they would often jackknife around as soon as the $\mathbf{S}+$ bar started to move and well before any other visual or auditory cue occurred. But a more direct test was in order.

\footnotetext{
Method

Subjects, Apparatus, and Preliminary Training. Six hooded males, about 140 days of age, constituted the bar-movement control group. The apparatus and preliminary training were as in Experiment 2.

Observational training. Three rats learned the go/no-go discrimination with the light above the right bar going on and the faint sound of a microswitch click as the $\mathrm{S}+$ (no bar moved) and with the sound of a sharp blow (click) on the base of the back panel directly behind the point where the tip of the left bar would ordinarily strike the floor as $S-$ (no bar moved). For the other three rats, S+ and S- were reversed, and no bar moved. Testing was the same as in Experiment 2, and if a subject did not press either bar within 5 min after entry to the test chamber it was removed. This procedure had never been necessary in previous experiments. Because our interest lay only in determining whether the moving bars were critical to the original observational learning of pressing the $S+$ bar, the subjects in Experiment 4 were not given reversal training.
}

\section{Results and Discussion}

The bar movement controls learned the go/no-go discrimination in a mean of 212.5 trials, about the same number as the subjects trained with moving bars. But their test trial behavior was markedly different. For the first time on test trials, including the initial one, the rats often failed to respond within 5 min after entry to the test chamber, and the latencies when barpressing occurred were also long. The median latency was $136 \mathrm{sec}$; for six hooded rats trained with moving bars in Experiment 2, the median was $49 \mathrm{sec}$. Also, when there was a failure to respond and when an S- barpress was counted as an error, exactly $50 \%$ of the test trials of the controls were incorrect. Thus, it appears that moving bars were critical to observational learning of pressing the $\mathrm{S}+$ bar. This finding makes it difficult to explain barpress learning noncognitively-on the basis of incipient approach to $\mathrm{S}+$ while watching from the tunnel and accidentally pressing the $S+$ bar during test trials.

\section{GENERAL DISCUSSION}

Probably the most interesting aspect of these results is that a small amount of S-R learning appears to predominate over a considerable amount of S-S learning, at least as we interpret it. According to the elicitation framework (Denny, 1971; Denny \& Adelman, 1955), reversal failure after testing is not unexpected because during observational reversal training in the tunnel the rat is not learning any response that directly competes with a particular barpress response in the unique stimulus situation of the test chamber. In an autoshaping context, when rats are permitted to make a competing response in the same situation in which learning occurred (Davey, Oakley, \& Cleland, 1981), they do, in fact, reverse.

The similarity to autoshaping and sign tracking (Hearst \& Jenkins, 1974) has already been noted, and it stands repeating. But there seem to be certain differences: (1) The learned response appears full blown, occurring on the initial test trial; it just does not facilitate the learning of the operant as in autoshaping of blocked responses (Browne, 1976). (2) The nature of the learned response seems to be specified by the nature of the S+ (moving bar), although considerably more research is needed to establish this point. (3) The learned response, once it is made, is never associated with reinforcement, yet it persists (Experiment 3). (4) There is a critical two-choice test phase. (5) When the rat presses the $\mathrm{S}+\mathrm{bar}$, it does not attempt to get back in the tunnel to obtain a food pellet; the rat presses the bar rather "deliberately" and just stays there, often hanging onto the bar. This last point invites the speculation that the rat is acting as if it were commenting on the environment, telling us in its own way that this is the $S+$ bar.

\section{REFERENCES}

Browne, M. P. The role of primary reinforcement and overt movements in autoshaping in the pigeon. Animal Learning \& Behavior, 1976, 4, 287-292.

Davey, G. C., OAkley, D., \& Cleland, G. G. Autoshaping in the rat: Effects of omission on the form of the response. Journal of the Experimental Analysis of Behavior, 1981, 36, 75-91.

Dé Russo, J. E. Observational learning of discriminative avoidance in hooded rats. Animal Learning \& Behavior, 1975, 3, 76-80. 
Denny, M. R. One bar-press per day: Acquisition and extinction. Journal of the Experimental Analysis of Behavior, 1959, 2, 81-85.

DENNy, M. R. Relaxation theory and experiments. In F. R. Brush (Ed.), A versive conditioning and learning. New York: Academic Press, 1971

Denny, M. R., \& Adelman, H. M. Elicitation theory: I. An analysis of two typical learning situations. Psychological Review, 1955, 62, 290-296.

Dodwell, P. C., \& Bessant, D. E. Learning without swimming in a water maze. Journal of Comparative and Physiological Psychology, 1960, 53, 422-425.

Groesbeck, R. W., \& Duerfeldt, P. H. Some relevant variables in observational learning of the rat. Psychonomic Science, $1971,22,41-43$.

Guthrie, E. R. The psychology of learning (rev. ed.). New York: Harper \& Row, 1952.

HeArst, E., \& Jenkins, H. M. Sign-tracking: The stimulusreinforcer relation and directed action. Austin, Tex: Psychonomic Society, 1974.

Jacobson, J. M., \& Sisemore, D. Observational learning of a lever pressing task. Southern Journal of Educational Research, 1976, 10, 59-73.
Kohn, B., \& Dennis, M. Observation and discrimination learning in the rat: Specific and nonspecific effects. Journal of Comparative and Physiological Psychology, 1972, 78, 292-296.

LAJore, J., \& Bindra, D. An interpretation of autoshaping and related phenomena in terms of stimulus incentive contingencies alone. Canadian Journal of Psychology, 1976, 30, 157-173.

LAshley, K. S., \& MCCARThY, D. A. The survival of the maze habit after cerebellar injuries. Journal of Comparative Psychology, 1926, 6, 423-433.

Solomon, R. L., \& Turnea, L. H. Discriminative classical conditioning in dogs paralyzed by curare can later control discriminative avoidance responses in the normal state. Psychological Review, 1962, 69, 202-219.

Spence, K. W., \& LippiTt, R. An experimental test of the signGestalt theory of trial and error learning. Journal of Experimental Psychology, 1946, 36, 491-502.

Tolman, E. C. Purposive behavior of animals and men. New York: Appleton-Century, 1932.

(Manuscript received May 21, 1982;

revision accepted for publication February 22, 1983.) 